\title{
Study on Optimal Adjustment Method on Stroke of Digital Beam Pumping Unit Based on the Change of the Indicator Diagram Area
}

\author{
Xiangqian $\mathrm{Xu}$, Haobin Zhou and Longlong Yin \\ Xi'an Shiyou University, Xi’an 710065, China
}

\begin{abstract}
The new optimal adjustment method on stroke of the digital pumping unit is proposed in this paper after analysis the main disadvantages of the existing digital pumping unit on adjustment speed. The new method is based on the change of the area of the indicator diagram. According to the change of the area of the diagram, the stroke of the pumping unit is optimized in the optimization module. The digital optimization module is set up between the RTU and the frequency converter of the existing digital pumping unit. The dynamic data compression algorithm is used for real-time access on the module, which improves the real-time performance of the system. And the module has the function to communicate with the control with the RTU and the frequency converter. So this pumping unit has the intelligent ability to control pumping speed, and no longer relies on the host computer. Tests about the module had been carried out at the oil production site and the changes of the indicator diagram and the adjustment of the stroke was recorded. The experimental results have shown that the method is feasible. The optimization method on stroke of the digital pumping unit is in accordance with the changing trend of the supply capacity of oil well.
\end{abstract}

Keywords—beam pumping unit; indicator diagram; parameter optimization

\section{The Current Adjustment Method On Stroke OF DIGITAL BEAM PUMPING UNIT}

The beam pumping unit is still one of the major oil recovery equipment in China [1]-[2]. In the process of building digital oilfields, the conventional pumping unit has been digitally transformed to have some digital and informational functions. And it is abbreviated as digital pumping unit. Digital pumping unit has become one of the major equipment for the digital construction of oilfields. The digital pumping unit is that the beam pumping unit is integrated with the oil well parameter acquisition module, sensor, control module and control device to realize the collection and upload of parameters of the operating status of the pumping unit or the functions of remote control or pumping parameter adjustment.

The wellhead RTU (remote transmission unit) can collect some information such as load, displacement, voltage, current and so on through sensors or the acquisition module. And the RTU can control the start and stop of the pumping unit through the start stop module, adjusts the balance of the beam by the balance motor and adjusts the stroke by the frequency converter. The wellhead RTU uploads data information and receives control instructions through the network communication equipment of the wellsite. Based on this control system, the way to choose the reasonable impact is to obtain the pump indicator diagram through the ground indicator diagram, and to obtain the liquid production according to the pump indicator diagram. The ground indicator diagram is the load displacement diagram, which is obtained by the information of load and displacement sensor. The equation of sucker rod system in the depth of the pump to the bottom of the well is solved by using the ground indicator diagram and the oil well parameters. So the load and displacement of the oil pump can be obtained and the pump indicator diagram can be obtained. The computer will give some conclusions, such as the oil well supply in insufficient according to the indicator diagram. According to these conclusions, the reasonable stroke of pumping unit is decided. Therefore, the water supply capacity of the oil well is constantly changing with the mining time, and the adjustment of the pumping unit needs to be carried out continuously.

The calculation method of the current digital oil pumping unit is complex and spends long time to calculate some data and takes up large memory in the industrial computer. The main reason is that the wave equation is required in the calculation process. And the software of "power diagram analysis" is to obtain the liquid yield of the oil well, which must be obtained by the ground indicator diagram after solved the wave equation to get the pump indicator diagram.

In this way, the closed loop control is formed in theory, which can solve the stroke optimization of pumping unit, but the real time of the closed loop control is poor, mainly because of the storage and reading of the indicator diagram data. The oil field mainly uses the relational database to store and read the data, not the real time database. Using a relational database for real-time data processing, the processing speed is much slower than the real time database. The real time database is charged according to the number of data points, and the cost is high. Suppose that every half hour, every well needs to collect a dynamometer map data. For some oilfield companies, the amount of data is very large, not only will occupy a lot of storage space and network bandwidth, but also more importantly generate high cost.

Therefore, the optimal adjustment method on stroke of the digital pumping unit is put forward according to the change of the area of the real time indicator diagram. The indicator diagram data is stored in the wellhead equipment by using a dynamic data compression method. 


\section{The Optimal Adjustment Method On Stroke OF The Digital Beam Pumping Unit Based On The Change OF INDICATOR DIAGRAM AREA}

\section{A. Adjustment methods on stroke of the pumping unit}

The pumping unit stroke is one of the parameters of swabbing, and there are many methods for its optimization and adjustment. The commonly used methods for optimizing swabbing parameters include the empirical, API recommended and S.G. Gibbs methods. The experiential method is based on the empirical formula of the pumping unit manufacturer to optimize the pump and rod string, but the well condition has large changes and the error is larger. The methods recommended by API are mainly compared with various diagrams, and sometimes it is not convenient to use in engineering [3]. The S.G. Gibbs method is mainly realized by solving the wave equation. Many scholars and engineers have done a lot of work about this. Ruidian Lv used the method of induction polynomial equation to optimize the parameters such as stroke, impulse, pump diameter and pump depth [4]. Shengli Zhang analyzed the sensitivity and influence of efficiency from 22 parameters, including equipment, work system and management and so on, and optimized it [5]. Shimin Dong established the dynamic parameter simulation model of the variable frequency control beam pumping unit, and optimized the real time frequency [6].

The experience method, the API recommended method and the S.G. Gibbs method in the swabbing parameter optimization method should be used to design the swabbing parameters of the oil well. In recent years, most of the methods put forward by many scholars rely on a lot of engineering data. But these algorithms are difficult to achieve on the wellhead RTU because of limited data storage space and other problems.

On the basis of the existing digital oil pumping unit, this paper puts forward a method of adjusting the stroke on the basis of the change of the area of the indicator. On the basis of the existing scheme, a modified optimization module is added. The module is set up between the wellhead RTU and the frequency converter of the current digital pumping unit. The main functions are to receive the load displacement information, to form the hanging point indicator diagram, to calculate the area and the change rate of the indicator diagram, to complete the control of the RTU to the frequency converter and to adjust and optimize the pumping speed. The functions that how to form the indicator diagram with load displacement sensor information and the communication control of the frequency converter are not described in detail here. This paper mainly introduces how to adjust the pumping speed according to the change of indicator diagram area.

The area calculation of indicator diagram is to be completed step by step based on the indicator diagram of information recording process. The hanging point indicator diagram is a graph consisting of a straight line connected by point-to-point, whose points are made up of displacement and load data points. Therefore, the indicator diagram is divided into the area calculation units according to the information acquired by the displacement information when calculating the indicator diagram area, which are the area calculation units formed by the up stroke process and the abscissa of the indicator diagram, and the area calculation units formed by the down stroke process and the abscissa of the indicator diagram. The area surrounded by the up stroke process and the indicator diagram abscissa is obtained by adding the area of the various units from the pumping unit "bottom dead center" to "top dead center" and calculating the area of each unit. In the same way, the area surrounded by the down stroke process and the indicator diagram abscissa is also obtained by adding the area of the various units from the pumping unit "top dead center" to "bottom dead center" and calculating the area of each unit. The area of the indicator diagram is calculated by the area surrounded by the up stroke process and the indicator diagram abscissa is subtracted from the area surrounded by the down stroke process and the indicator diagram abscissa.

The area of the current indicator diagram $S_{S_{k}}$, the area of the next time indicator diagram $S_{k+1}$ and the area of the previous time indicator diagram $S_{k-1}$ are recorded using in the above method. The difference between the area of the current indicator diagram and the next time indicator diagram shown as $\Delta_{k}=S_{k}-S_{k+1}$, and the difference of the area of the previous time indicator diagram and the area of the current indicator diagram shown as $\Delta_{k-1}=S_{k-1}-S_{k}$, are respectively calculated. If $\Delta_{k}$ is in the range of the error tolerance, the current pumping speed is kept constant and the current pumping speed is the best. Otherwise, comparing the size of the two differences between $\Delta_{k}$ and ${ }_{\Delta_{k-1}}$, if the error is greater than the allowable range $\left(\Delta_{k}>\Delta_{k-1}\right)$, you need to reduce the pumping speed; if not $\left(\Delta_{k} \leq \Delta_{k-1}\right)$, you need to increase the pumping speed. Whether it is to improve or reduce the pumping speed, the decision of the best pumping speed is $\Delta_{k}$ within the allowable range of the error tolerance. The specific judgment process is shown in Figure 1. 


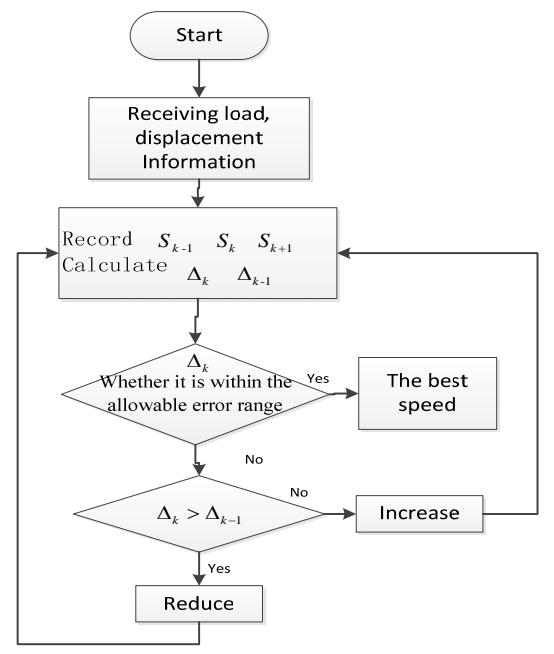

FIGURE I. THE PROCESS DIAGRAM OF OPTIMIZATION AND ADJUSTMENT

\section{B. Lossless compression algorithm for the indicator diagram}

In order to realize real-time optimal adjustment on stroke of the digital beam pumping unit according to the area change of the indicator diagram, it is necessary to access the dynamometer map in real time. If it is collected and accessed in the way of collection and access, it will need larger storage space, which is difficult to be realized on the RTU of the wellhead. Therefore, the data compression method of the indicator diagram is used for storage. Jinnuo Li's lossless compression algorithm of indicator diagram is applied to carry out the lossless data compression of the suspension indicator diagram of the beam pumping unit in the small storage space embedded chip, by comparing and analyzing a large number of dynamometer map data and graphics.

The core of the data compression algorithm is to compress by finding repetitive or regular data, mainly divided into two kinds, which are lossy compression and lossless compression methods [7]-[9]. When the lossy compression algorithm is used to compress the data, the original data can not be fully recovered. This method reduces the amount of data by sacrificing some quality and improves the compression ratio [10]. And it is often used on the Internet, especially in the field of streaming and telephone. The lossless data compression [11] is the reconstruction of the compressed data, and the reconstructed data is exactly the same as the original data. The lossless compression data is not lost after compression, and the compressed data can be completely restored before compression.

After analyzing the mainstream data compression algorithms in detail, Jinnuo Li [12] proposed a dynamic data compression method based on the repeatability of adjacent data different in indicator diagrams. The data compression method of the indicator diagram of Jinnuo $\mathrm{Li}$ is used to compress the data. The concrete steps are as follows:

1) Clear the wrong collection of data and determine the accuracy of the data. Usually there will be some rough data in the process of data acquisition, so these data must be deleted for the efficiency and accuracy of data compression. Then the accuracy of the indicator diagram data is processed according to the needs of the project.

2) Obtaining the data difference of the indicator diagram. The difference between two adjacent values from left to right is calculated by the set DV (DV, Different Value). The repeated difference data are recorded, represented by the set NDV (NDV, Normal Different Value). The special difference data are recorded, represented by the set SDV (SDV, Special Different Value). SDV is the set of DV and NDV difference sets.

3) These data are stored separately according to the different attributes of SD, NDV, and SDV, as shown in Figure 2. The first byte stored source data SD (SD, Source Data), then the number of storage of special SDV value, and then each special difference position and data value, according to the difference in the storage of normal NDV, each of which is the first sign of normal value.

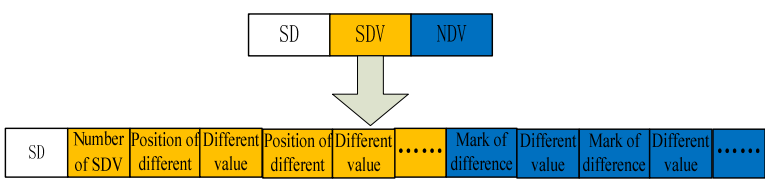

FIGURE II. STORAGE ALGORITHM

4) Repeat steps (1)-(3) compress the other indicator diagrams, according to the optimization and adjustment method.

5) Update of indicator diagram data. The new indicator diagram is used for compression and storage instead of the old indicator diagram.

During the oil recovery process, the capacity of the oil supply is constantly changing, and the indicator diagram is constantly changing. Therefore, during the uninterrupted oil extraction process of the pumping unit, the compression storage and reading of the repetitive indicator diagram need not be interrupted.

\section{The realization of the optimization module of the stroke adjustment}

From the above analysis, it can be concluded that the input of the optimization and adjustment module is the load sensor and displacement sensor information collected by RTU, the output is the control information for the frequency converter. And then, the load sensor is arranged in the position of the rope hanging device of the pumping unit. The displacement sensor is chosen as the angle displacement sensor, which is installed on the beam supporting shaft of the pumping unit. The frequency converter is arranged in the intelligent control cabinet of the pumping unit. The optimization module of pumping speed adjustment completes the corresponding function of the program using MCU, and the microcontroller model is C8051f020.

\section{1) Receiving load displacement information}

The single-chip microcomputer C8051f020 can achieve MODBUS communication. And the RTU is using the standard MODBUS communication protocol. Therefore, the microcontroller C8051f020 and the RTU can be carried out 
data communication based on MODBUS. The RTU supports RS485 bus, and MODBUS communication is also based on this bus. So, according to the communication protocol, the single-chip C8051f020 can read the data from the RTU that upload to the industrial PC. There are two serial communication unit in C8051f020, the sending pin (TXD) of one serial communication unit to connect the pin 4 of the SP485 chip, the receiving pin (RXD) of the same one serial communication unit to connect the pin 1 of the SP485 chip, and the pin 6 and pin 7 of the SP485 chip connect to the 485 bus. The author has written a serial communication program, which can read the information load and displacement sensor in function.

\section{2) The change of indicator diagram area and stroke adjustment}

According to the information of the load displacement, the area calculation of the indicator diagram is established in the RAM area of the single chip C8051F020. Using the method of the previous section, the coordinate system is established inside the single chip, and the area of each information point is accumulated, and the area of the single indicator diagram is obtained. And then, the area of the current indicator diagram, the area of the next time indicator diagram and the area of the previous time indicator diagram are calculated, and their differences are calculated. Taking into account the different oil wells, pumping unit models will be different, so in the calculation of the power diagram area, the data will be normalized. So it is convenient to calculate the area of indicator diagram and its change trend. The trend of the change of the indicator diagram area is compared, and the direction of adjusting the stroke is obtained.

\section{3) Frequency converter communication}

The communication control must be established between the single chip C8051F020 and the frequency converter in order to send the command from the single chip to the frequency converter to adjust the stroke. Now there is the MODBUS communication between the RTU and the frequency converter. So the corresponding communication program is written in C8051f020, keeping its communication mode unchanged. The communication control program of the frequency converter is programmed by another serial communication unit of C8051f020, and the hardware circuit is similar to the RTU.

\section{TEST VERIFICATION}

In order to verify the effectiveness of the adjustment method, an oil well is tested, and the pumping unit is CYJW7-2.5-26HF pumping unit. After the application of the stroke optimization module, the pumping unit can be adjusted automatically without stopping, and can be adjusted automatically. From the results recorded, we can see that when the stroke is higher, the area of indicator diagram becomes smaller, and the pump fullness may not be high. This shows that the pumping speed and capacity for the liquid oil does not match. When the stroke is lower, the area of indicator diagram is larger and the fullness of the pump may be improved. With the continuation of oil production, and the continuous adjustment and optimization of the stroke, the changes in the indicator diagram area will be very small. Finally, the changes in the range of allowable error, the best state is reached.

\section{CONCLUSIONS}

Through the above theoretical analysis and experimental verification, the conclusions are as follows:

1) It is convenient and feasible to adjust and optimize the stroke based on the change of the indicator diagram area. On the basis of the existing digital oil pumping unit, the optimization module is adjusted and the intelligence of the pumping unit is increased.

2) The application of the dynamic data compression algorithm of indicator diagram makes the optimization module of the stroke adjustment to run in the small capacity chip.

\section{REFERENCES}

[1] Daokai Yang, Haiyun Chen, Ning Ji. Design of Frequency Conversion Control System of Long Stroke Pumping Unit without Beam, Automation Application 1(2016), 12-17.

[2] Xiangqian Xu, Haobin Zhou, Mao Li, Vector Control System of Beam Pumping Unit with Load-adaptive Function, Science \& Technology Review 30(2012) ,22-26.

[3] Chaoqin Tan. An overview of optimal design method for production of oil pumping wells [J]. China Petroleum and Chemical Industry. 2012, (8):37-39.

[4] Ruidian Lv, Yuansheng Zhang, Shimin Dong. Optimal design of pumping well system [J]. Southwest Petroleum Journal.1993, 15 (3): 91-96.

[5] Shengli Zhang, Jimin Guo, Weilan Chen. Optimization design and application based on parameter sensitivity analysis of pumping well [J]. Journal of Oil and Gas Technology. 2008, 30(5):321-323.

[6] Shimin Dong, Weicheng Li, Xiaofang Zhao. Dynamic simulation and real-time frequency optimization of a variable frequency beam pumping unit [J]. China Mechanical Engineering. 2016, 27(12):1585-1590.

[7] Sayood K. Introduction to data compression [M]. 4th ed. Hongfeng Jia, trans. Beijing : Posts \& Telecom Press. 2014:1-138.

[8] Lenan Wu. Data compression [M]. 3rd ed. Beijing: Publishing House of Electronics Industry. 2012:1-7.

[9] Hilbert M, López P. The world's technological capacity to store, communicate, and compute information [J]. Science. 2011, 332(6025):60-65.

[10] Written I H, Bell T C, Moffat A, et al. Semantic and generative models for lossy text compression [J]. The Computer Journal. 1994, 37(20): 83-87.

[11] Chanda P, Elhaik E, Bader J S. HapZipper: sharing HapMap populations just got easier[J]. Nucleic Acids Research. 2012, 40(20): 59.

[12] Jinnuo Li, Renbin Gong, Qun Li. A lossless data compression and storage method of pump dynamometer [J]. Acta Petrolei Sinica. 2016, 37(2): 266-273. 